\title{
8. Re-imagining Digital Care and Health
}

\begin{abstract}
In Chapter 8 we turn to Co-futuring Kinship — the ways in which past and present practices inform how the future of the kinship for care at a distance. This is particularly important for "super-aging" contexts like Japan in which one in three is of 80 years old. Chapter 8 sets the picture for discussion around digital health in which mobile media is fully imbricated in. Discussions around a "silver bullet" in the form of a mobile app still dominate despite the fact that there is much work into the need for social, rather than technological, solutions.
\end{abstract}

Keywords: aging; digital health; mobile apps

In this section of the book we explore the role of mobile social media practices as a vehicle for quotidian, mundane notions of digital health. Rather than conceiving digital health as something that is designed by technologists and biomedical scientists for others, we consider the important role of the lived experience of everyday users to create their own forms of health and wellbeing in, and through, digital contexts. As surgeon Atul Gawande notes, aging in Western contexts has often been simplified through a medical lens - instead, Gawande argues that aging needs to be understood as a more holistic social and cultural phenomenon, where it is about finding meaning and purpose throughout all of life's transitions (2014). Understanding aging as part of a continuum, in which intergenerational unidirectional sociality is constantly at play, is key to Co-futuring Kinship.

Indeed, Co-futuring Kinship requires us to reflect upon the ways in which the digital creates and affords different forms of care, continuity and connection. We deploy the concept of co-futuring as a way to emphasize our participants' role in designing the contextual uses of digital media in everyday life. Co-futuring, as defined by UNESCO chair Sohail Inayatullah, is a design process that allows participants to relate differently to the past and present (2008). Inayatullah defines six different ways to think about

Hjorth, L., K. Ohashi, J. Sinanan, H. Horst, S. Pink, F. Kato, B. Zhou, Digital Media Practices in Households: Kinship through Data. Amsterdam: Amsterdam University Press, 2020 DOI 10.5117/9789462989504_CHO8 
co-futuring as a way to collaborate with different stakeholders to investigate future solutions for sustainability and social inclusion. Co-futuring sees participants as co-designers in their futures as informed by their past and present practices. Co-futuring puts lived experience at the center of the participatory process. In the case of growing datafication of life in which citizens can feel disempowered by everyday corporate or governmental surveillance, co-futuring allows for more micro and more intuitive forms of activism.

The notion of co-futuring became prevalent in our ethnography over the three years. Over that time, relationships and digital media dynamics changed and evolved. Elements such as tracking and automation became heightened in conversations and practices. As datafication became increasingly prevalent through mobile devices in everyday life_-from intentional self-tracking to more un-intentional forms of surveillance by corporations and governments - we needed ways in which to not only reflect on the impact of these technologies but also the implications on our future lives. For example, what happens to our data when we die? Many of our participants were only starting to think about this and many didn't have digital legacy plans in their wills.

In this final section the book turns more to reflection on present practices as future-in-making-co-futuring. We returned to our participants once more to gain their insights into how digital media-especially in terms of locative and mobile media-were starting to take on new dimensions. In particular, we focused on aging and the role of mobile media for informal notions of health and care. We discussed with both our participants as well as including older adult family members about their relationships to health and care in an age of datafication. Were technologies helping to enhance certain aspects of health? Was the technology camouflaging bigger issues about intergenerational care?

We begin this chapter with a discussion of mobile games in relation to quotidian digital health. We then reflect upon the complex and often contradictory space of applification and datafication alongside hearing our participants' valuable insights. By connecting practice with co-futuring approaches we hope to reconnect lived experience with hopes and desires for a future in which technologies are embedded within the social.

\section{Mundane Mobile Games as Quotidian Digital Health}

We met with Machiko, an 86-year-old woman who lived in Kyoto. When her husband died a decade ago, Machiko started going to her local gym, 
as a way to make friends and focus on her health and wellbeing. While she made some good friends, over the past decade Machiko found that some of her friends had passed away, while others had increasing mobility issues. Machiko felt less motivated to go to the gym when her friends weren't there.

One day she went to the local park with her friend Koko and Koko's grandson, Haru. Haru asked Koko and Machiko if they could play with him. He guided them around the park showing them the digital overlays of the Pokémon GO universe. They had fun playing. Haru asked Koko if she might play it often and so they could play in parallel. Machiko offered to play with Koko to help. After a few months, Koko and Machiko started to feel that they understood the game and would often go for a walk to achieve steps for fitness whilst also playing Pokémon GO.

Since its highpoint in 2016, Pokémon $G O$ has begun to attract older players as a way to avoid social isolation and increase physical activity. In suburban areas of Kyoto, it is not uncommon to see players over the age of 60 slowly navigating their neighborhoods through the augmented Pokémon $G O$ interface. In a country with a large and growing aging population, Japan is now facing a future in which people will increasingly age and die alone. In places like Singapore, the elderly has also embraced Pokémon $G O$ as a way to keep fit and socialize, while also opening up opportunities to play and interact meaningfully with younger generations.

But Asia is not alone. In locations like Badalona, Spain, social workers are teaching older adults who live alone to play Pokémon $\mathrm{GO}$, and engage with others by joining local Pokémon GO communities of players (Hjorth and Jimenez 2019). Here, young and old play together in urban spaces. What becomes apparent is that there are ways of addressing various aging and health issues that are more effective than medication or new-fangled technological devices. Rather, through the now mundane and habitual medium of the mobile phone and a somewhat hackneyed three-year old mobile game - which is old for a mobile game - significant improvements to quality of life can be achieved. Pokémon $G O$ transforms urban space into something playful and social.

Ethnographic exploration of these lived experiences highlights the need for digital health to focus more carefully on the social dimensions of aging well rather than just having a "silver bullet" solution in the form of yet another mobile app. Instead, we can learn a lot from "old" media like Pokémon $G O$ and how they can be redeployed and reinvented in ways that demonstrate the importance of understanding social practice in designing for digital health solutions-especially in terms of aging well. 
Here Pokémon GO signals a few future directions for mobile games.

Firstly, mobile games afford both ambient and located social connection, and so can be used to counteract the emotional and physical isolation experienced by many people-young and old - in playful and innovative ways. Secondly, they can facilitate connections between younger and older generations through an interface that has mutual significance and enjoyment for both, creating the possibility for social change. Thirdly, they move across public and private spaces in ways that can usefully link informal and formal systems and routines of care, especially as we move towards aging societies.

Fourthly, mobile games also provide us with ways to understand the complex relationality between humans and our companion species, as we form intimate bonds through playful interaction, share sensory and affective modes of perception and proprioception, yet are distinct in our embodied uses of media. Fifthly, in an age of Big Data and gamification, and an associated focus on the quantitative and utilitarian aspects of technology, the sensory intimacy and ambient sociality of mobile games and playful apps force us to attend to the complex tapestry of media use, and the corresponding ethnographic and interpretive adaptability required of researchers.

The rise of Pokémon GO illustrates the importance of haptic digital media in affording space for the non-normalized body. Haptics allow players with impairments like hearing or vision to be able to participate in meaningful ways. The rise of digital media in terms of multisensorial dimensions such as haptics — which marries media and sensory study approaches—will be increasingly important as we move towards aging and super-aging societies (that is, over a third of the population is over 65 years). In the next section we contextualize this phenomenon in relation to debates around mobile applification and datafication. We then turn to the concept of feeling data. This discussion provides context for Chapter 9 in which we discuss participants' practices and perceptions.

\section{Applified and Datafied: Quantified Self and Digital Health}

Since 2010, the significance of Big Data, and the response to it through forms of the QS movement, has been unmistakable (Kennedy et al. 2016). As danah boyd and Kate Crawford (2011) note, much of the debates around Big Data need to acknowledge that no matter how "big" the data, it is always subjective. Data questions are riddled by the searchers' own perception and thus inherently human in their scope. Moreover, they argue the need for 
transparency, and access to the ways in which algorithms shape definitions of society. For ethnographers such as Dawn Nafus, big data, like little data, is about storytelling (Nafus 2016; Nafus and Neff 2016).

As digital health experts such as sociologist Deborah Lupton (2016) have noted, datafication has created a double edged sword for health-selftracking offers insight and a space for reflection about one's own exercise and yet, this same reflective data can be used by corporations in ways we are still only just understanding in light of, for example, the recent Cambridge Analytica debacle. For Natasha Dow Schüll, wearable technology allows for the designing of "self care" (2016) which has paradoxical dimensions. She argues:

key artifacts in a new cultural convergence of sensor technology and selfcare that I call 'data for life', wearables are marketed as digital compasses whose continuous tracking capacities and big-data analytics can help consumers navigate the field of everyday choice making and better control how their bites, sips, steps and minutes of sleep add up to affect their health. By offering consumers a way to simultaneously embrace and outsource the task of lifestyle management, I argue, such products at once exemplify and short-circuit cultural ideals for individual responsibility and self-regulation (cited in Ruckenstein and Schüll 2017, 317).

As aforementioned, concepts like applification and datafication have very much become part of quotidian urban everyday life with the saturation of smartphones. Datafication is the process whereby our personal data is given over to platforms for their own interests and financial benefit (van Dijck 2015). In the rise of datafication-i.e. where data saturates seemingly every aspect of our everyday lives - there have been two key camps. One camp stems from the QS movement founded by Wolf and Kelly in 2007 which sought to see individuals "gamify" their life in creative ways (Gilmore 2016; Lupton 2016b; Nafus and Sherman 2014) such as Strava and fit bit. In this space, approaches such as ethnography have blossomed to explore the multiple overlays of the digital in relation to the senses, sociality and movement through everyday places and spaces (Hjorth et al. 2017). This area explores what Jason Farman has called "social proprioception" (2011) — whereby the knowledge of the lived body and its movement is focused upon in the context of sociality. The other camp sees the darker side of neo-liberalism whereby data is being co-opted by corporations in ways that compromise privacy-what has been called dataveillance (Ruppert 2011; van Dijck 2015; Lupton 2016). 
Datafication and algorithmic cultures are now commonplace-that is, where data penetrate, invade and analyze our daily lives, causing anxiety and seen as potentially inaccurate statistical captures (Hjorth and Hinton 2019). These tensions and the paradoxes of power/powerless; tactic/strategy; identity/anonymity are highlighted through the lens of artists "playfully resisting" through creative projects that scrutinize the normalization ("bellcurving") of QS and datafication usage at a broader and everyday sociopolitical level. For some, self-tracking and QS allows for a better sense of the body - a deeper proprioception. Through thinking about the body as "laboratory," alternative human-centerd (and "more-than-human") social digital methods emerge around ethnography and critical making. But for others, the emphasis on numbers and quantification goes against real understanding of practice as something that is story-based and nuanced and can't be summed up by numbers (Lupton 2016; Humphreys 2018).

In general use applification is an IT industry term used to refer to one way of simplifying and making complex software and processes easier to use. Applification can be seen in the emergence of apps on the mobile device (van Dijck 2017). Applification, as part of "listening devices," raise important questions around privacy and surveillance and the use of the data by government, corporations and individuals. And while QS advocates will argue that mobile apps allow for the democratizing of data and tools for cartography and tracking, there are counter arguments about the deployment of data in ways we are yet to fully realize. This is especially in the case when user's die and they don't have a digital legacy provision in their will — thus affording corporations like Facebook to own their data, much to the horror of bereaved loved ones.

On mobile devices, apps are the embodiment of the ideal of applification. Fitness tracking apps like Strava (used mainly for bicycle riding and running) are indicative of how self-tracking mobile apps (as part of the broader QS movement) are playing a powerful role in people's lives. In these apps, one user's exercise becomes part of others' movements, allowing the data overlays about place-making to be shared as a social act. As users become more playful with their use of data and digital media, the role of feeling data, through complex multisensorial ways of being in the world becomes heightened.

\section{Feeling data}

The rise of self-tracking QS measures on mobile media allow for reflection-what some scholars have called feeling the data (Lupton 2017). Lupton has been working in the space of digital health for over a decade now. More 
recently her work has explored the role of what digital health "feels" like (2017). As Lupton notes, feeling the data can be understood as a process in which movement, cartography, data and place-making coalesce into a heighten sense of social proprioception.

This discussion raises one of the key tropes in current game studies around what a game "feels" like. Game designers such as Swick have highlighted that unpacking the often-tacit notion of the game "feel" is crucial to understanding player engagement (2008). Game phenomenologists such as Keogh have explored the role of game feel as part of the proprioception of the knowing, playing body (2018). Indeed, as important as "feeling" might be to the game or medium's affect, it has often been untheorized. Understanding and articulating the feelings associated around media and data requires complex multisensorial methods to unpack these experiences.

As Lupton identifies, more work into understanding the multisensorial dimensions of spaces and places is needed - in sum, the affective atmospheres (2017). As a term developed in cultural geography by Gernot Böhme (and especially mobility studies), it explores the affective and emotional attunements of humans and more-than-humans as they move through spaces and places. In areas like phenomenological psychopathology it is being deployed to understand the importance of atmospheres in helping with client consultation and related treatment (Costa et al. 2014). Focusing on affective atmosphere puts the lived experience at the center. As Lupton notes:

Affective atmosphere is understood as an assemblage of affect, humans
and nonhumans that is constantly changing as new actors enter and leave
spaces and places. Affective atmospheres are shaped by their multisensory
properties: how spaces and places are physically encountered via their
visual, haptic, aural, olfactory and taste properties is central to the feelings
they generate. Affective atmospheres can have profound effects on the ways
in which people think and feel about and sense the spaces they inhabit
and through which they move and the other actors in those spaces $(2017,1)$.

As Lupton argues, there is very little research into the "concept of affective atmospheres to the context of health, illness and healthcare" $(2017,2)$. With the rise of haptic screens through smartphones, the role of the digital as an embodied part of the everyday life comes to the forefront. Haptic media studies coalesce key areas: sensory studies, new materialism and new qualitative non-media centric approaches. It highlights the need to develop language around multisensorial approaches in and around haptics which emphasise the importance of the lived body and social proprioception. 
The rise of haptic media and trying to understand the phenomenon through practice has led to many subareas of ethnography including multisensorial ethnography (Pink 2009), tactile ethnography (Pink et al. 2016) and haptic ethnography (Richardson and Hjorth 2017). In particular, haptic ethnography seeks to coalesce the work done around mobile media in terms of the material, sensory and corporeal aspects (Richardson and Hjorth 2017). This process requires deploying methods and techniques that give voice to experience through multisensorial elicitation.

As Richardson and Hjorth note, haptic technologies such as smartphone screens are impacting our sensorial experience of being-in-the-world, beingwith-others and being-with-media (2019). Informed by and informing this approach, many researchers are increasingly focusing on the intimate, social and playful nature of mobile touchscreens. The role of haptic technologies is especially important when it comes to vulnerable agencies - that is, elderly, disabled or more-than-human users. Haptic technologies allow for multisensorial approaches to media that encourage different lived experiences and skillsets. Haptic screens re-focus our attunement away from the primacy of the visual and aural towards other sensorial knowledges. In this way, haptic screens require us to develop different techniques and methods in our ways of understanding the world.

At the intersection of the QS movement and gamification (Deterding et al. 2011), mobile media and its attendant apps are providing new ways in which to think about creativity, play and labor in everyday life (Hjorth 2018). From body measuring devices such as fitbits and apps to measure sleep, one could argue that everyday life is being colonized by datafication. However, in practice we see a diversity of resistance and subversives that suggest that not all of life is quantifiable (Neff and Nafus 2016; Hjorth 2018). We see how existing sociocultural practices and the ways in which users can queer the applification of everyday life through play and creativity. Indeed, far from just a medium for beautiful and young people, Instagram, like its audiences, are diversifying. Audiences are getting older and alternative, non-normative modes of body and performativity are being celebrated (Olszanowski 2014; Tiidenberg 2014).

\section{Conclusion}

In the next chapter we explore the complex and alternative ways in which the data can be felt by participants in quotidian contexts. We explore the practices and perceptions around data and caring intergenerationally. How does mobile and locative media provide particular forms of care? What 
problems around care does it highlight? Understanding the rise of mobile apps and self-tracking has become a key area of emerging research (Fox and Duggan 2013; Lupton 2016, 2017; Fox 2017). We begin with Japan as an example of a super-aging society which is grappling with its own cultural understandings of intergenerational care and digital health, then turn to our participants to explore their own experiences and reflections. We reflect upon rituals and perception as a process of co-futuring present practices.

\section{References}

boyd, danah, and Crawford, Kate. 2011. "Six Provocations for Big Data." In A Decade in Internet Time: Symposium on the Dynamics of the Internet and Society, September. https://papers.ssrn.com/sol3/pape"s.cfm?abstract_id=1926431. Accessed 10 February 2018.

Costa Cristina, Carmenates, Sergio, Madeira, Luís, and Giovanni Stanghellini. 2014. "Phenomenology of atmospheres. The felt meanings of clinical encounters." Journal of Psychopathology 20: 351-357. https://www.jpsychopathol.it/issues/2014/ vol20-4/SOPSI4-14.pdf\#page=12. Accessed 5 April 2018.

Fox, Nick. 2017. "Personal health technologies, micropolitics and resistance: a new materialist analysis." Health 21 (2): 136-153. DOI: 10.1177/1363459315590248.

Fox, Susannah, and Duggan, Maeve. 2013. "Tracking for Health," Pew Research Center: Internet and Technology, 28 January. https://www.pewinternet.org/ 2013/01/28/tracking-for-health/. Accessed 4 March 2018.

Gawande, Atul. 2014. Being Mortal. New York: Metropolitan Books.

Hjorth, Larissa. 2018. "Ambient and soft play: Play, labour and the digital in everyday life." European Journal of Cultural Studies 21 (1):3-12. DOI: 10.1177/1367549417705606. Hjorth, Larissa, and Sam Hinton. 2019. Understanding Social Media. Second Edition. London: Sage.

Humphreys, Lee. 2018. The Qualified Self: Social Media and the Accounting of Everyday Life. Cambridge, MA: The MIT Press.

Inayatullah, Sohail. 2008. "Six pillars: futures thinking for transforming." Foresight 10 (1): 4-21. DOI: 10.1108/14636680810855991.

Kennedy, Helen, Thomas Poell, and José van Dijck. 2015. "Introduction: data and agency." Big Data \& Society 2. DOI: 10.1177/2053951715621569.

Lupton, Deborah. 2016a. "The diverse domains of quantified selves: selftracking modes and dataveillance." Economy and Society 45 (1): 101-122. DOI: 10.1080/03085147.2016.1143726.

—.2016b. The quantified self: A sociology of self-tracking. Cambridge; Malden, MA: Polity Press. 
-2017a. "Feeling your data: Touch and making sense of personal digital data." New Media \& Society 19 (10): 1599-1614. DOI: 10.1177/1461444817717515.

_.2017b. "How does health feel? Towards research on the affective atmospheres of digital health." Digital Health 3. DOI: 10.1177/2055207617701276.

Nafus Dawn. 2016. Quantified:Biosensing Technologies in Everyday Life. Cambridge, MA: The MIT Press.

Nafus, Dawn, and Gina Neff. 2016. Self-Tracking. Cambridge, MA: The MIT Press.

Nafus, Dawn, and Jamie Sherman. 2014. "This one does not go up to 11: The Quantified Self Movement as an Alternative Big Data Practice." International Journal of Communication 8 (1): 1791-1793.

Olszanowski, Magdalena. 2014. "Feminist Self-Imaging and Instagram: Tactics of Circumventing Sensorship." Visual Communication Quarterly 21 (2): 83-95. DOI: $0.1080 / 15551393.2014 .928154$.

Pink, Sarah. 2009. Doing Sensory Ethnography. London: SAGE Publications.

Pink, Sarah, Jolynna Sinanan, Larissa Hjorth, and Heather Horst. 2016. "Tactile Digital Ethnography: Researching Mobile Media through the Hand." Mobile Media \& Communication 4 (2): 237-251. DOI: 10.1177/2050157915619958.

Pink Sarah and Vaike Fors. 2017. "Being in a mediated world: self-tracking and the mind-body-environment." Cultural Geography 24 (3): 375-388. DOI: 10.1177/1474474016684127.

Ruppert Evelyn. 2011. "Population objects: Interpassive subjects." Sociology 45: 218-233. DOI: 10.1177/0038038510394027.

Schüll, Natasha Dow. 2016. "Data for life: wearable technology and the design of self-care.” BioSocieties 11 (3): 317-333. DOI: 10.1057/biosoc.2015.47.

—.2017. Our metrics, ourselves. Public Books, 26 January. https://www.publicbooks.org/our-metrics-ourselves/. Accessed 2 March 2018.

Sharon, Tamar. 2017. "Self-tracking for health and the Quantified Self: re-articulating autonomy, solidarity, and authenticity in an age of personalized healthcare.” Philosophy \& Technology 30 (1): 93-121. DOI: 10.1007/s13347-016-0215-5.

Richardson, Ingrid, and Larissa Hjorth. 2019. "Haptic play: Rethinking media cultures and practices." Convergence 25 (1):3-5. DOI: 10.1177/1354856518815275. _.2017. "Mobile media, domestic play and haptic ethnography." New Media \& Society 19 (10): 1653-1667. DOI: 10.1177/1461444817717516.

Ruckenstein, Minna, and Natasha Dow Schüll, 2017. "The Datafication of Health.” Annual Review of Anthropology 46: 261-278. DOI: o.1146/annurevanthro-102116-041244.

van Dijck, José. 2014. "Datafication, dataism and dataveillance: big data between scientific paradigm and ideology." Surveillance \& Society 12: 197-208. 